\title{
Unravelling Belgian Blue cattle farmers' adoption intention towards diagnostic tools: Integrating insights from behavioural economics and socio-cognitive theories
}

\author{
Carla Mingolla $^{\mathrm{a}, *}$, Wouter van $\mathrm{Mol}^{\mathrm{b}}$, Liselot Hudders ${ }^{\mathrm{c}}$, Veroline Cauberghe ${ }^{\mathrm{a}}$, \\ Edwin Claerebout ${ }^{\mathrm{b}}$ \\ ${ }^{a}$ Department of Communication Sciences, Ghent University, Belgium, Korte Meer 7-9-11, 9000, Gent, Belgium ${ }^{1}$ \\ ${ }^{\mathrm{b}}$ Department of Virology, Parasitology, Immunology, Faculty of Veterinary Medicine, Ghent University, Belgium, Salisburylaan 133, B-9820, Merelbeke, Belgium \\ ${ }^{\mathrm{c}}$ Dept. of Communication Sciences, Dept. of Marketing, Ghent University, Belgium, Korte Meer 7-9-11, 9000, Gent, Belgium
}

\section{A R T I C L E I N F O}

\section{Keywords:}

Theory of planned behaviour

Behavioural biases

Mange

Macrocyclic lactones resistance

Farmers' adoption of diagnostic tools

Focus group studies

\begin{abstract}
A B S T R A C T
Macrocyclic lactone resistance in Psoroptes ovis mites, causing psoroptic mange, is emerging in beef cattle. Therefore, diagnostic tools that can indicate macrocyclic lactone resistance should be implemented at farms in order to control these parasitic infections and slow down emerging resistance. Unfortunately, the adoption of such tools remains relatively poor. This study explores which beliefs, and behavioural biases that misconstrue these beliefs, underlie farmers' adoption intention. Five semi-structured focus groups were conducted with Belgian Blue cattle farmers $(n=38)$. Inductive analysis revealed that farmers' positive attitudes towards acaricides use and risk perception towards acaricide resistance made them rather reluctant to implement diagnostic tools. In addition, farmers' susceptibility to acaricide resistance occurring on their farm was weakened by the optimism and availability bias. Deductive analysis revealed that economic reasons, usefulness of the diagnostic tool and contribution to animal wellbeing motivated farmers to adopt such tools (i.e., behavioural beliefs). However, the loss aversion and time discounting bias weakened farmers' beliefs related to economic reasons. The veterinarian was seen as the responsible actor for implementing diagnostic tools, while colleague-farmers' opinion related to the diagnostic tool was also valued (i.e., normative beliefs). The latter belief was strengthened by the bandwagon-effect bias. Farmers' beliefs about economic costs related to the diagnostic tool tended to hinder adoption intention, while the veterinarian's assistance motivated farmers to implement such tools (i.e., control beliefs). The loss aversion bias also strengthened farmers' control beliefs related to the diagnostic tools' perceived costs. As such, this article provides more insights into the (ir)rational factors shaping farmers' intention to adopt diagnostic tools. These insights might help animal health organisations to design communication strategies to stimulate the adoption of diagnostic tools on beef cattle farms.
\end{abstract}

\section{Introduction}

Psoroptic mange in cattle is caused by the Psoroptes ovis ( $P$. ovis) mite. Psoroptic mange is a major problem in the beef cattle industry, affecting the Belgian Blue breed in particular (Blutke et al., 2015; Sarre et al., 2012, 2015). This parasitic disease has a negative effect on animals' welfare and growth, leading to important production losses (Doherty et al., 2018; Sarre et al., 2015). Three groups of acaricides are frequently used to control psoroptic mange: macrocyclic lactones, amitraz and synthetic pyrethroids (flumethrin). Importantly, regarding mange treatment, there is no legislation in Belgium; the country where the study was conducted. When animals show clinical signs (e.g., intense pruritus and thick scabby lesions; Stromberg et al., 1986), farmers are responsible for contacting their veterinarian, who in turn provides them with chemical acaricides and advice on how to use those acaricides. Chemical acaricides can thus only be bought through the veterinarian. Farmers themselves execute the treatment of mange. Information on how to optimally treat mange can be found on the website of Animal

\footnotetext{
* Corresponding author.

E-mail addresses: Carla.Mingolla@UGent.be (C. Mingolla), Wouter.vanMol@UGent.be (W. van Mol), Liselot.Hudders@UGent.be (L. Hudders), Veroline. Cauberghe@UGent.be (V. Cauberghe),Edwin.Claerebout@UGent.be (E. Claerebout).

1 Affiliation where the research was conducted.
} 
Health Care Flanders (Dierengezondheidszorg Vlaanderen) ${ }^{2}$.

Since the 1980s, macrocyclic lactones have been considered one of the most effective treatments for controlling psoroptic mange because of their high efficacy and ease of use (Lifschitz et al., 2018). However, after many years of intensive use and often incorrect treatment implementation (e.g., underdosing, suboptimal formulations), resistance of $P$. ovis to macrocyclic lactones is emerging in beef cattle (Lekimme et al., 2010; Lifschitz et al., 2018; Sarre et al., 2012; van Mol et al., 2020). This developing resistance raises considerable concerns for mange control and highlights the need to implement diagnostic tools to control parasitic infestations in Belgian Blue cattle on farms (Doherty et al., 2018). Such diagnostic tools can inform farmers whether their cattle are infested with $P$. ovis mites and whether the mites are resistant to macrocyclic lactones. Based on those results, farmers can adopt the correct treatment course. Specifically, when the tool indicates macrocyclic lactone resistance, farmers should switch to other acaricides that may be more effective. Implementing such tools will eventually lead to a more targeted use of acaricides (i.e., less frequent and intensive and more sustainable use of acaricides), slow down the emerging macrocyclic lactone resistance and accordingly resulting in sustainable mange control.

Generally, farmers (and veterinarians) are responsible for implementing preventive measures against diseases on their farms. The adoption of diagnostic tools thus requires a behavioural change in farmers. In practice, however, these behavioural changes seem rather difficult to accomplish (Speksnijder and Wagenaar, 2018). For example, despite published advise on how to treat mange properly (on the website of Animal Health Care Flanders), Sarre et al. (2012) showed that less than half of Belgian Blue cattle farmers who participated to the study ( $n$ $=680$ ) applied an optimal treatment scheme. In order to successfully implement diagnostic tools on beef cattle farms and change the behaviour of farmers, a thorough understanding of the factors underlying farmers' intentions to adopt diagnostic tools is necessary. These insights can be used by policy makers to develop effective communication strategies to facilitate the uptake of diagnostic tools on beef cattle farms (Jansen and Lam, 2012; Ritter et al., 2017; Speksnijder and Wagenaar, 2018).

Several socio-psychological models have been used to predict and understand the factors underlying human behaviour and behavioural change. One of the most widely used theories in the research of human behaviour is the Theory of Planned Behaviour (TPB;Ajzen, 1991). The TPB suggests that behavioural decisions are the result of a reasoned process in which behavioural intention is determined by attitudes towards behaviour, subjective norms and perceived behavioural control. Attitude is defined as an individual's favourable or unfavourable evaluation of the behaviour. Subjective norms refer to perceived social pressure towards the behaviour. Finally, perceived behavioural control is the personal assessment of the feasibility of executing the behaviour in a given context. These determinants are influenced by beliefs towards the desired behaviour, which refer to salient beliefs. Ajzen (1991) distinguishes three types of beliefs, each affecting the determinants of the TPB: behavioural, normative and control beliefs. Behavioural beliefs refer to the perceived (dis)advantages of performing a certain behaviour; normative beliefs are a person's subjective beliefs that a particular important referent wants the individual to perform a given behaviour; and control beliefs are related to various factors that hinder or facilitate a certain behaviour, such as time or costs (Ajzen, 1991). Thus, the underlying beliefs, which are called indirect predictors, indirectly explain behavioural intention (Yuriev et al., 2020). Behavioural intention, in turn, is a proxy for actual behaviour (Ajzen, 1991).

Recently, the TPB has been used in the agricultural domain to

\footnotetext{
${ }^{2}$ Animal Health Care Flanders is a non-profit organisation in Flanders that helps livestock farmers and veterinarians in the monitoring, tackling and prevention of animal diseases on farm level).
}

understand farmers' conservation behaviour and their intention to adopt conservation agriculture practices (e.g., Bagheri et al., 2019; Borges et al., 2016; Hyland et al., 2018). It has also been used to investigate farmers' intentions to adopt practices related to animal welfare and health, such as the adoption of environmental enrichment to improve the welfare of farmed pigs (Borges et al., 2019), the prudent use of antimicrobials in livestock (e.g., Jones et al., 2015; Speksnijder and Wagenaar, 2018), adoption intention of diagnostic tools to control for gastrointestinal nematode (GIN) infections in dairy cattle and to understand farmers' business decisions (da Silva et al., 2020; Daxini et al., 2018).

Despite the general usefulness of the TPB model to predict and understand human behaviour, the model has received some criticism (Alarcon et al., 2014; Conner and Sparks, 2005; Yuriev et al., 2020). Firstly, a major limitation of the TPB is that the model assumes that behaviour is based on a rational and systematic decision-making process, which implies a high level of cognitive effort (Alarcon et al., 2014; McLeod et al., 2015). The TPB therefore offers less insight into the irrational aspects of human behaviour which are driven by habits, emotions, biases, impulsivity, etc. (Conner and Sparks, 2005; Dessart et al., 2019; Huijps et al., 2010; McLeod et al., 2015; Wolf, 2017). Secondly, the model is not a holistic model and effects of other influencing variables are ignored. However, Ajzen (1991) describes the model as flexible and open to further expansion if important proximal determinants are identified (Bagheri et al., 2019; Yuriev et al., 2019). Consequently, in the agricultural domain, several studies have included additional variables into the model, such as moral norms, knowledge (e.g., Bagheri et al., 2019; Rezaei et al., 2018), self-identity (e.g., Borges et al., 2019; van Dijk et al., 2016), perceived severity and susceptibility (e.g., Rezaei et al., 2019) to increase the explanatory power of the TPB. Despite the integration of these additional variables to the model, most of these variables are also rational variables and thus do not take into account other irrational factors affecting farmers' behaviour (Rezaei et al., 2018; Wolf, 2017). The current study will further examine how these irrational factors may misconstrue the formation of beliefs related to the adoption intention of diagnostic tools.

A growing body of literature illustrates the potential of behavioural economics to gain a better insight into farmers' behaviours (Dessart et al., 2019; Huijps et al., 2010; Mingolla et al., 2019; OECD, 2012; Wolf, 2017). Neoclassical economic models suggest that farmers act rationally when full information is available to them, always strive to maximise profit and implement measures with a well-thought-out cost-benefit analysis (Frederiks et al., 2015); Huijps et al., 2010; Wolf, 2017). However, farmers' behaviour is hardly ever fully rational, and there are also other irrational but predictable factors that influence their decision making (Wolf, 2017). According to theories of behavioural economics, human decision making often deviates from making optimal and fully deliberate choices (i.e., rationality), and there are certain behavioural biases and other irrational tendencies in human decision making (Camerer et al., 2004; Mullainathan and Thaler, 2000). A behavioural bias is assumed to be a systematic reasoning error in the outcomes of decisions or judgements individuals make that occurs when individuals are processing and interpreting information in their environment. 'Systematic' refers to the fact that these biases return in individuals' behaviour (individuals thus tend to show the same behavioural biases; Korteling et al., 2018). For example, imagine that you have to decide which job is more dangerous - police officer or lumberjack. If you quickly think of multiple examples of police shootings or news report about policemen dying, you will (falsely) believe that being a police officer is more dangerous, while in fact statistics show that lumberjacks are more likely to die on the job. This availability bias, whereby individuals tend to estimate the frequency of future events based on the ease with which examples come to mind, influences our judgement and decisions (Kahneman et al., 1982). Behavioural biases can also be defined as misperceptions of reality, which refers to the mismatch of our judgement and reality (Korteling et al., 2018; Samson, 2016). 
In the agricultural domain, the influence of behavioural biases upon farmers' adoption of environmentally sustainable practices ${ }^{3}$ has often been acknowledged (e.g., Dessart et al., 2019; He et al., 2019; OECD, 2012). In animal health-related behaviours, some authors have emphasized the relevance of behavioural biases to farmers' decision making (Huijps et al., 2010; Jansen and Lam, 2012; Wolf, 2017). For example, Huijps et al. (2010) empirically explored the influence of a behavioural bias, the loss aversion bias, upon farmers' decision making. This bias suggests that individuals have a tendency to prefer avoiding losses over acquiring equivalent gains. The authors demonstrated that penalty systems (i.e., monetary penalty for not changing behaviour) are more effective than bonus systems (i.e., monetary rewards for behavioural change) in inciting farmers' intention to adopt management measures at improving udder health (e.g., wearing milking gloves).

Notwithstanding the emerging importance of behavioural economics, this discipline does not form a substitute for rational behavioural change models (e.g., socio-psychological or neoclassical economic models). Instead, it complements these models by offering interesting insights into individuals' (mis)perceptions and the emotiondriven factors that may lead to sub-optimal decisions (Wolf, 2017). This assumption is supported by Dessart et al. (2019), who suggest that biases might explain why and how behavioural factors (including cognitive factors such as perceived risk, social factors such as injunctive norms and dispositional factors such as personality) influence farmers' adoption of environmentally sustainable practices. Farmers' decisions to adopt new diagnostic tools may occur less frequently, often have long-term economic consequences and may involve an investment. Thus, farmers' decisions to adopt a diagnostic tool can be expected to be somewhat controlled and well thought out (i.e., a rational consideration). However, as previously discussed, this does not mean that farmers always make well-thought out decisions based on rational considerations, nor that these decisions and rational considerations are free from biases (Dessart et al., 2019; Wolf, 2017). Consequently, when trying to understand and predict farmers' intention to adopt a diagnostic tool, both rational and irrational factors should be taken into account.

Accordingly, Mingolla et al.'s (2019) study integrated insights from behavioural economics into the TPB framework to predict Belgian Blue cattle farmers' adoption intention for diagnostic tools. In particular, four behavioural biases were integrated into the model as biased beliefs. ${ }^{4}$ The results of a survey administered to Belgian Blue cattle farmers revealed the influence of four biased beliefs on farmers' adoption intention through the concepts of attitude, subjective norms and perceived behaviour control (PBC): availability bias, loss aversion bias, bandwagon effect bias and default bias. First, the results showed that farmers' beliefs about the opinion of significant others towards the diagnostic tool affected both attitude and subjective norms. This irrational behaviour of "following the herd" instead of using one's own information is also referred to as bandwagon-effect bias (Samson, 2016). Second, the results indicated that perceived cost in effort and time of using such a tool negatively affected attitudes and PBC towards the diagnostic tool. These perceptions of costs can be distorted due to the loss-aversion bias.(Huijps, 2010; Wolf, 2017). Third, farmers tendency to retain to their current treatment positively affected farmers' PBC. This positive impact of default treatment can be explained by the default bias, which suggests that individuals have a strong tendency to retain the status quo

\footnotetext{
${ }^{3}$ Environmentally sustainable practices are farming practices whose main goals are associated with conserving water, reducing the use of fertilizers and pesticides and promoting biodiversity in ecosystems. Examples of such practices are conservation management and organic farming practices.

${ }^{4}$ As previously discussed, the predictors of the TPB framework - attitude, subjective norms and PBC - are determined by salient beliefs (i.e., behavioural beliefs, normative beliefs and control beliefs). In their study, the authors explored whether these beliefs are biased through the principles of behavioural economics, hence biased beliefs.
}

or follow pre-set options because the disadvantage of changing behaviour is perceived as greater than the advantage (e.g., time-cost versus more effective treatment). Therefore, people tend to resist change even when the alternatives provide more benefits (e.g., financially beneficial; Samson, 2014; Wolf, 2017). Farmers were inclined to retain their default treatment and perceived themselves to be highly in control of the decision to treat mange on their farms. To conclude, the results showed that prevalence of mange in farmers' herd significantly affected subjective norms. Thus, the higher the perceived prevalence of mange, the more valuable the opinions of important others about the treatment of mange were perceived to be. These perceptions of prevalence of mange may have affected their estimation of future mange problems, a bias that is referred to as the availability bias (Kahneman et al., 1982). The three concepts of the TPB influenced adoption intention positively, with subjective norms being the strongest predictor followed by attitude and PBC.

Mingolla et al.'s (2019) study was a first step towards complementing the TPB framework with insights from behavioural economics to explore the irrational and rational factors affecting Belgian Blue cattle farmers' adoption intention regarding diagnostic tools. However, their study does not provide in-depth insight into the beliefs underlying the three constructs of TPB (and thus indirectly underlying behavioural intention) or how biases might distort (or misconstrue) these beliefs (i.e., biased beliefs). These insights are important and can help provide more specific advice for the development of communication strategies to stimulate the uptake of diagnostic tools. In particular, for the behavioural beliefs concept, it is important to elicit the perceived advantages and disadvantages of adopting a diagnostic tool (e.g., animal welfare, economic outcomes, etc.; Borges et al., 2019). For the concept of normative beliefs, it is important to elicit who social influencers (i.e., important referents) are to Belgian Blue cattle farmers (Sok et al., 2015). For the concept of control beliefs, it is important to reveal the factors that might facilitate or impede the adoption of a diagnostic tool such as time and costs (Speksnijder and Wagenaar, 2018). Importantly, a number of biases (amongst which are the behavioural biases identified in the conceptual model of Mingolla et al., 2019) might misconstrue these beliefs. Therefore, it is also important to identify which biases misconstrue these beliefs and how (Dessart et al., 2019).

Consequently, the objectives of this qualitative study are twofold: 1) to elicit farmers' beliefs related to adopting diagnostic tools on Belgian Blue cattle farms and 2) to elicit which biases distort these beliefs underlying farmers' adoption intention and how they do so. Given the complexity of the decision to change treatment programs and the interdependency of various factors (e.g., behavioural factors), we used a qualitative research method to gain richer insight into the perceptions of the factors that appeared to hinder and motivate Belgian Blue cattle farmers' adoption intention of diagnostic tools that can lead to more sustainable use of acaricides.

\section{Materials and methods}

Aiming to respond to our two objectives, the current study used the focus group methodology amongst Belgian Blue cattle farmers $(N=38)$. Focus group methodology is a form of qualitative research in which a group of people (usually six to 12 individuals) is interviewed by one or more facilitators or interviewers. This type of interview research is useful because it uses group interaction to stimulate discussion and allows participants to exchange perspectives about a certain topic. These different perspectives provide a more thorough understanding of participants' underlying motivations, beliefs and perceptions for a particular behaviour (Kitzinger, 1995).

\subsection{Recruitment of participants}

Data was collected during the months of February through April 2016. Five focus groups were held in five different provinces in Flanders, 
Table 1

Descriptives of the focus group participants.

\begin{tabular}{lll}
\hline Conditions & Levels & $\#$ \\
\hline Farm type & Beef & 38 \\
Sex & Female/male & $1 / 37$ \\
Age & Min & 31 \\
& Max & 64 \\
& Mean & 55 \\
\% Prevalence mange on farm & No mange (0\%) & 4 \\
& Moderate (1-20\%) & 22 \\
& Much (>20\%) & 10 \\
& Missing & 2 \\
Province located & West Flanders & 7 \\
& East Flanders & 7 \\
& Antwerp & 3 \\
& Limburg & 10 \\
Farm size & Flemish Brabant & 11 \\
& Small ( $\leq 149$ animals) & 23 \\
& Large ( $\geq 150$ animals) & 14 \\
Used acaricides & Missing & 1 \\
(multiple choices possible) & Macrocyclic lactones & 31 \\
& Amitraz & 20 \\
& Synthetic pyrethroids & 3 \\
& Missing & 5 \\
\hline
\end{tabular}

Table 2

Initial topic list of the focus groups studies.

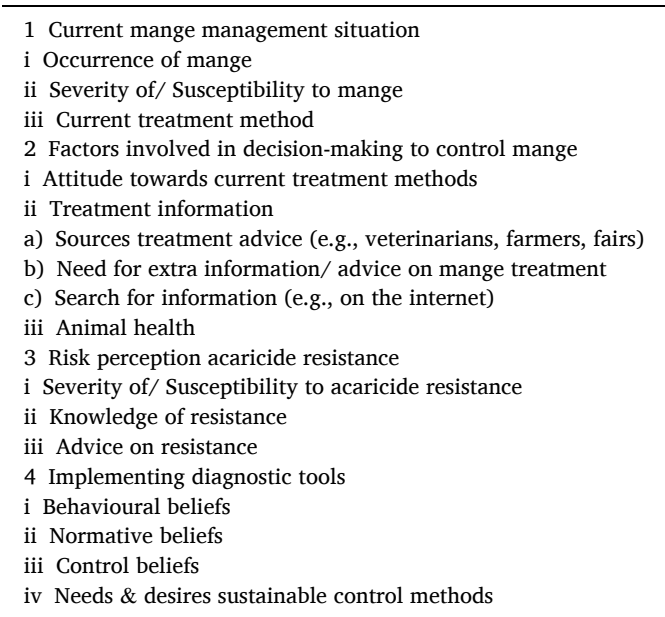

Belgium (East Flanders, West Flanders, Antwerp, Flemish Brabant and Limburg; see Table 1) and were moderated by three interviewers - one social scientist and two veterinary scientists. The interviewers/moderators were the two veterinary scientists (second authors and last author) and the minute secretary was the social scientist (first author). All focus groups were audio recorded, and recordings were transcribed and analysed by the first author. All interviews were conducted in Dutch; therefore, quotes presented in this paper are translated as literally as possible and crosschecked by the authors.

Via the monthly e-newsletter of Animal Healthcare Flanders, which possesses the most complete and up-to-date list of Belgian Blue cattle farmers in Flanders, farmers were asked to participate in a focus group study related to the topic of "mange". Besides being a beef cattle farmer, no further specifics were required. Farmers who replied to the invitation received a follow-up invitation containing information about the time and place of the focus group in their region. In total, 38 farmers participated in the focus group study. The focus groups lasted between 45 and $91 \mathrm{~min}$ (recorded time), with an average of $65 \mathrm{~min}$.

\subsection{Topic list}

The focus group studies were conducted with a semi-structured pattern. Semi-structured interviews tend to generate less response bias compared to structured interviews or questionnaires (Furnham, 1986). A number of topics were defined a priori, based on the theoretical constructs from the TPB framework (Ajzen, 2002) and on the conceptual model from Mingolla et al. (2019). In particular, the semi-structured interview questions were designed to gain insights into the salient beliefs underlying the determinants of the TPB framework (attitude, subjective norms and $\mathrm{PBC}$ ) and the behavioural biases that might distort these beliefs. Topics are presented in Table 2.

We started with introductory questions about farm specifics (e.g., severity mange, occurrence of mange). The second part was aimed at gaining a general understanding of the factors involved in the farmers' decision-making process in controlling mange. The third part focused on exploring farmers' perceptions about the emerging acaricide resistance and the perceived risk of acaricide resistance occurring on their farm. The final part focused on identifying the beliefs underlying farmers' adoption intention regarding new diagnostic tools to improve the effectiveness of mange treatment (i.e., sustainable use of acaricides) and how such tools should be optimised (e.g., needs and desires for diagnostic tools). Throughout the focus groups, close attention was given to potential biases of participants, on which the researcher probed more indepth.

Table 3

Template containing 5 socio-cognitive concepts that underlie farmers' intention to adopt diagnostic tools, complemented with insights from behavioural economics (i.e., behavioural biases) to better understand how these concepts are being formed.

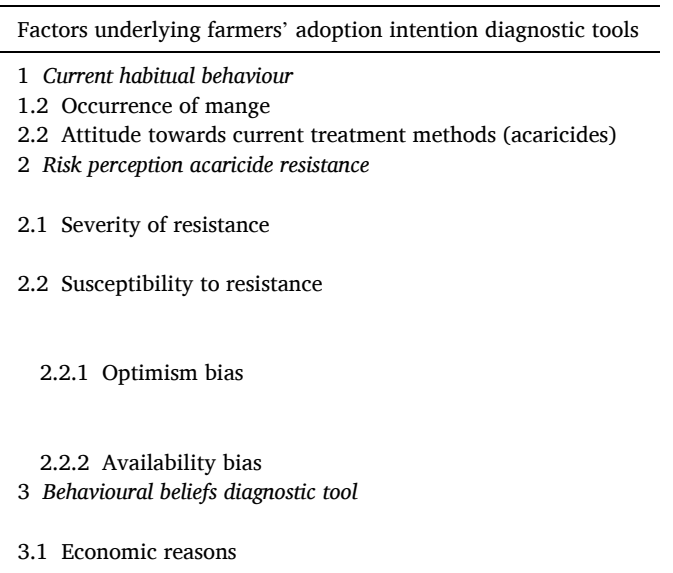

3.1.1 Loss-aversion bias and time-discounting bias

3.2 Perceived usefulness

3.3 Animals' wellbeing

4 Normative beliefs diagnostic tool

4.1 Veterinarians' role

4.2 Farmers' opinion and behaviour

4.4.1 The bandwagon-effect bias

4.2.2 Farmers' personality traits

5 Control beliefs diagnostic tool

5.1 Perceived economic costs

5.1.1 Loss-aversion bias

5.2 Veterinarians' assistance 


\subsection{Data analyses}

The general inductive approach was used as the analysis strategy, to move data between deduction and induction (Thomas, 2006). Deductive analysis refers to data analysis guided by prior assumptions or hypotheses identified by the researcher (Strauss and Corbin, 1994). Inductive analysis refers to new themes, concepts or biases generated by exploration and interpretation of the raw data. These two approaches are often used simultaneously (Thomas, 2006). Accordingly, in this study, deductive analysis took the factors identified in the conceptual model of Mingolla et al. (2019) into account - i.e., behavioural beliefs (attitude), normative beliefs (subjective norms), control beliefs (perceived behavioural control), availability bias, loss aversion bias, bandwagon effect bias and default bias. Inductive analysis brought forward habits in relation to current treatment behaviour (i.e., current habitual behaviour), risk perception of acaricide resistance, time discounting bias and optimism bias. Time discounting-bias refers to the fact that present rewards are weighted more heavily than future ones; i.e., short-term benefits are more valuable than long-term benefits. Optimism bias refers to the fact that people tend to underestimate the probability of negative events - for example, underestimating a certain risk (e.g., getting cancer) relative to other people (Shepperd et al., 2002).

The focus groups were manually transcribed verbatim, and field notes and codes were added. The first step of data analysis involved reading through all transcripts to understand the data as a whole. In the first phase of analysis, distinct words or sentences were given a label (e. g., 'In consultation with my veterinarian, then yes, I would implement a diagnostic tool' - veterinarian as important referent). In the second phase, labels that referred to similar content were grouped into themes (veterinarian as important referent - normative belief). For this phase, a topic frame was used based on the previous literature (i.e., deduction; Mingolla et al., 2019). Some labels were grouped outside the topic frame. For example, risk perception (e.g., 'I do not perceive my farm to be susceptible to resistance' - susceptibility resistance). These newly found labels were given a theme (e.g., susceptibility resistance - risk perception acaricide resistance) and added to the topic frame (i.e., induction). Consequently, the previously given themes were re-analysed, taking into account the newly found themes. Once all themes were established from the data, a conceptual framework (see Table 3) was developed in phase three. In this last phase, themes were translated into concepts underlying the adoption intention of diagnostic tools.

\section{Results}

The results are structured within the framework of the TPB, with its three beliefs underlying the adoption intention of diagnostic tools: behavioural beliefs, normative beliefs and control beliefs. Two additional dimensions are added to the framework: current habitual behaviour and risk perception of acaricide resistance. For each of these dimensions, the biases that were brought up during the focus groups are discussed in the discussion section. As such, there is a clear difference between facts (i.e., what did the farmers say) and the authors' interpretation of biases (i.e., why is this a behavioural bias) distorting different beliefs and other rational factors. See Appendix A, Fig. A1 for an overview of biases distorting the rational factors underlying adoption intention. The demographics for participants presented in each focus group are presented in Table 1.

\subsection{Current habitual behaviour}

Current habitual behaviour related to current treatment methods (i. e., use of acaricides) is formed by two concepts: occurrence of mange on farms and attitude towards current treatment methods. Most farmers (30 out of 38) reported having experienced problems with mange in recent years, and 23 out of 38 farmers perceived it to be a severe problem. Eleven of the 38 farmers indicated that the problem had worsened in recent times and had become more difficult to treat; e.g., 'I can say I have never had problems with mange until last year. Last year was the first time that mange occurred on my farm, and I think it is a severe problem, especially to get rid of it' (Focus group [FG] 5, Farmer 1, male). Regardless of whether there was a low or high incidence of mange on their farms, 33 out of 38 farmers argued that mange had become a recurring problem and that mange control had become a frequent activity due to the reduced efficacy of current treatment methods:

'The thing that bothers us is that mange can be kept under control, but it occupies you weekly. It really consumes a part of your time and is an unnecessary workload that should not have to be done if the products would work properly. '(FG 1, Farmer 4, male)

Some farmers (6 out of 38) argued that the disease had even occurred during summertime, when previously it usually only occurred during the autumn/winter season: 'We used to have problems with mange in winter, now it also happens in summer. I guess that's a new thing' (FG 5, Farmer 5, male). Only 4 out of the 38 farmers reported that mange did not often occur on their farm: 'I don't have severe problems with mange, I can keep it under control' (FG 5, Farmer 4, male).

There was a clear difference between farmers attitudes' toward the efficacy of macrocyclic lactones versus amitraz to control mange. Farmers' attitudes towards macrocyclic lactones were only moderately positive, as they appeared to be easy to use, but less effective: 'When I treat my cattle with [macrocyclic lactones], mange doesn't go away easily. You have to treat them at least twice' (FG 5, Farmer 3, male); 'The products [macrocyclic lactones] are less effective than before; I have to treat my cattle several times before mange goes away' (FG 4, Farmer 6, male). In contrast, farmers' attitudes towards amitraz were rather positive, since 32 out of 38 farmers were convinced that amitraz is the most effective way to treat mange: 'I treat my cattle by using [amitraz]. I used to use [macrocyclic lactones], but that is not effective anymore' (FG 2, Farmer 1, male); 'Without using [amitraz], you can't keep mange under control' (FG 2, Farmer 1, male). This was confirmed by another farmer who stated, 'When you use [amitraz], mange is gone. It can be kept under control' (FG 2, Farmer 2, male).

Notably, less preference was given to the usage of this effective acaricide because of the more intensive workload (animals need to be treated several times, and a whole-body treatment [washing, spray] is necessary): 'The reason that we do not always use [amitraz] is because it requires a lot of work' (FG 1, Farmer 3, male). However, about half of the farmers (20 out of 38 ) reported using amitraz when the macrocyclic lactones were no longer effective in treating their cattle or combining several treatment methods: 'I use [macrocyclic lactones], and when I see that an animal doesn't respond to it, I use [amitraz]' (FG 4, Farmer 5, male); 'I use [amitraz] and [macrocyclic lactones] at the same time' (FG 3, Farmer 4, male).

Given these rather positive attitudes towards acaricides, 25 out of 38 farmers believed that mange was still treatable and controllable. Some of these farmers (7 out of 38) also believed that good farm management facilitates the control of mange. For example, these farmers thoroughly cleaned their sheds during summer while their cattle were out to pasture, while other farmers preventively treated purchased animals before putting them in the sheds:

'I have problems with mange every year, but I am convinced that you can keep mange perfectly under control when you clean your sheds and treat your cattle three to four times per year. So, you can keep it under control for a while' (FG 5, Farmer 7, male).

\subsection{Risk perception acaricide resistance}

During the focus groups, reflections arose about risk of treatment failure (i.e., resistance of $P$. ovis to acaricides). These risk perceptions stem from farmers' perceptions about the severity of acaricide resistance and the susceptibility of their farm to acaricide resistance.

The majority of the farmers ( 22 out of 38 ) agreed that resistance to macrocyclic lactones is occurring more frequently and perceived it to be 
a serious problem: 'With a product like [macrocyclic lactones] that goes straight into the blood, shouldn't it help? After all those years that we have used [macrocyclic lactones], we can say that there has been an aggressive resistance during the past five years' (FG 1, Farmer 4, male); 'There is definitely resistance to [macrocyclic lactones], but not for [amitraz]' (FG 2, Farmer 1, male).

Nevertheless, even though these farmers were aware of the severity and urgency of resistance to macrocyclic lactones, 23 out of 38 farmers believed that their farm was not susceptible to resistance because of their belief in the efficacy of the other acaricides: 'At this moment, I don't see resistance as a threat to my farm because [amitraz and pyrethroids] are still effective to treat mange' (FG 3, Farmer 8, male); 'Resistance is not really a threat to my farm, because when I use [amitraz] mange is gone' (FG 2, Farmer 2, male). Furthermore, 8 out of 38 farmers merely perceived their farm would be susceptible to resistance in the future when all available methods fail to cure (which they believed was not yet the case): 'If all treatments lose their effectiveness, we would see resistance as a threat for the future, for the breed. But at this moment, I don't see resistance as a threat to my farm' (FG 3, Farmer 6, male).

Furthermore, although 30 out of 38 farmers indicated having experienced problems with mange on their farms, 18 out of 38 farmers agreed that adopting new tools to test for resistance would be especially interesting for farms with a high incidence of mange or for farms that endured major problems with mange: 'As long as only $2 \%$ of my cattle is infected with mange, I won't think about implementing a test. But for someone whose cattle are 30-40\% infected with mange, the test would be interesting' (FG 4, Farmer 5, male). In addition, these farmers endorsed the belief that one has to have many problems with mange before implementing new diagnostic tools that test for resistance: 'When aiming to implement a diagnostic tool, you have to have problems with mange on your farm, or else it's useless to use it' (FG 5, Farmer 4, male).

\subsection{Behavioural beliefs related to the diagnostic tool}

The focus groups brought forward three behavioural beliefs related to implementing diagnostic tools farms: economic reasons, usefulness of the diagnostic tool and animal wellbeing. In particular, farmers' beliefs related to the (dis)advantages of implementing diagnostic tools on their farms came to the surface.

Some farmers (12 out of 38) believed it important to invest in diagnostic tools for economic reasons: 'If you don't diagnose for resistance before applying a treatment, mange is treated ineffectively, and it will only cost you more money' (FG 3, Farmer 5, male). Some farmers ( 8 out of 38 farmers) also considered the implementation of the test as a return on investment in the long-run:

'The current products cost a lot of money. If the diagnostic test would allow you to fix the problem, you could earn back your money in the long-run. You have to dare to commit to this; it may cost more money in the short-run, but you can get a return on investment. You have to be proactive and do something that provides a solution.' (FG 3, Farmer 2, male)

However, when farmers reflected about investing in diagnostic tools, 17 out of 38 farmers raised questions about the profitability of such tools: 'When you develop a diagnostic tool, it will have financial consequences, no? For a farmer it has to be profitable' (FG 3, Farmer 4, male).

Perceived usefulness of diagnostic tools was believed to be a positive outcome of the diagnostic tool. The majority of the farmers (25 out of 38) agreed that the usefulness of a diagnostic tool is an important characteristic in the decision to adopt such a tool: 'All tools that can help are welcome. It may cost money, but it has to work. Something that works efficiently is worth its money' (FG 4, Farmer 5, male). When discussing the usefulness of the tool, some farmers primarily questioned the added value of implementing diagnostic tools on their farm: 'But what does the diagnostic test imply? Will it tell you which product to use or only that there is resistance on our farm?' (FG 4, Farmer 8, male).

A common argument amongst 8 out of 38 farmers was that they were already aware of resistance to some acaricides (especially macrocyclic lactones) based on their experience of treatment failure with the currently used products on their farm: 'To be honest, there is resistance because mange always comes back. I don't think the test is necessary to know whether there is resistance or not, because resistance is present' (FG 1, Farmer 3, male). However, when reflecting further about the usefulness of diagnostic tools, 10 out of 38 farmers reached a consensus about the fact that these tools could be useful if they provided an effective means of optimising the control of mange (and thereby decreasing labour) on their farms and preventing resistance from spreading. They also believed that using such tools would be useful in helping to prevent the development of resistance to all available methods (in addition to macrocyclic lactones):

'If the diagnostic test can indicate which product is ineffective, it can save us a lot of time by indicating resistance to that particular product. This would indicate that we have to change our treatment and would prevent us from treating mange ineffectively' (FG 1, Farmer 5, male).

'I think such a tool would be useful to prevent resistance developing for all available methods, because imagine the mites become resistant to all available treatments; what should we do then?' (FG 4, Farmer 6, male).

In deciding how to treat their cattle against mange, 27 out of 38 farmers also considered the health of their animals as an important factor. These farmers mentioned their concerns about the suffering of their animals (scratching, bleeding, etc.) resulting from a $P$. ovis infestation and declared that they would implement diagnostic tools to improve the wellbeing of the animals: 'The wellbeing of our animals is an important factor when considering adopting a diagnostic test. It is not enjoyable to see that your animal is suffering' (FG 1, Farmer 6, male). In addition, about half of these farmers (16 out of 38) also associated animal wellbeing with the economic profitability of their farm: 'It's all linked to each other. Animal health goes along with being profitable. When your animals are healthy, they will be more profitable' (FG 5, Farmer 8, male).

\subsection{Normative beliefs related to the diagnostic tool}

The focus groups brought forward two normative beliefs. In particular, veterinarians and farmer colleagues were believed to be the important referents who could place pressure on farmers to adopt diagnostic tools.

Most farmers (31 out of 38 ) considered their veterinarian to be the most important advisor for their farm and valued his/her opinion and advice above all else concerning mange control. Accordingly, farmers' current treatment schemes were based on their veterinarians' advice and opinions: 'Veterinarians are the base for everything' (FG 1, Farmer 5, male); 'Primarily, information about treatment methods comes from my veterinarian; he proposes a scheme, and I start following it' (FG 3, Farmer 1, male). Furthermore, 33 out of 38 farmers indicated that they would listen to the advice of their veterinarian when he/she recommended implementing new treatment methods. Particularly, these farmers argued that the veterinarian knew their farm best, so any decision about change of treatment should be made in consultation with the veterinarian: 'I don't think I would use a diagnostic test on my own. In consultation with my veterinarian, then yes, I would implement it. But implementing a test on my own initiative, I don't think so' (FG 2, Farmer 2, male). Notably, 25 out of 38 farmers emphasized that followup advice from their veterinarian should be linked to implementing new diagnostic tools on farms: 'There has to be some advice linked to the diagnostic tool. What's the point otherwise? You know there is resistance, so what? There has to be something additional to the test' (FG 2, Farmer 5, male).

Besides the veterinarians' opinion and advice, 19 out of 38 farmers also cared about the opinions of other farmer colleagues about the 
diagnostic tools, as they stated they would implement a new diagnostic test when their colleagues had implemented it successfully: 'I would wait for other colleagues to have tried out the test before I would implement it' (FG 5, Farmer 9, male); 'If in practice the diagnostic test proves to be effective, you can't stay behind; you have to follow the herd' (FG 3, Farmer 8, male). However, 12 out of 38 farmers indicated that they would implement such methods without awaiting a try-out by their peers: 'I would be willing to use the diagnostic test before my colleagues implement it. Somebody has to start first' (FG 3, Farmer 4, male).

Despite veterinarians' important role regarding advice on treating mange on farms, there was reportedly a lack of communication about resistance between farmers and their veterinarians. Particularly, the majority of the farmers (30 out of 38) reported not having received any information from their veterinarian about resistance:

'We do not get informed about resistance. We already discussed that we need to use [amitraz and pyrethroids] amongst colleagues because all other products fail to cure, but we did not really consider the causes of this treatment failure and the role of resistance in this matter. (FG 2, Farmer 2, male)

This view was reiterated by others: 'When you speak about treatment failure with the veterinarian, he will simply give you another product' (FG 1, Farmer 6, male); 'Although I would like to receive some information about emerging resistance, we don't receive any information from our veterinarian' (FG 2, Farmer 3, male).

\subsection{Control beliefs related to diagnostic tools}

Focus groups brought forward two control beliefs that could impede and facilitate the adoption of diagnostic tools: perceived economic costs and the veterinarians' assistance.

It was obvious that the perceived economic costs were prevalent in farmers' thoughts when considering adopting diagnostic tools. The majority of farmers (20 out of 38 ) believed economic costs of the diagnostic tool to be an important factor that might prevent the implementation of such tools. 'I think most farmers would use the diagnostic tool if the costs are not too high' (FG 4, Farmer 2, male); 'Depends on what the diagnostic tool would cost; if it is reasonable, than I would maybe consider implementing a diagnostic tool on my farm' (FG 2, Farmer 1, male).

The focus groups also revealed that 18 out of 38 farmers believed their veterinarian should perform the test: 'The execution of the test should be done in a proper manner, so I believe the veterinarian should perform the test' (FG 2, Farmer 1, male); 'I don't think I would implement a diagnostic tool on my own initiative; my veterinarian would have to perform the test' (FG 3, Farmer 4, male). However, 17 out of 38 farmers stated that they would use the new test themselves. Veterinarians' lack of time and economic reasons were given as main argument to perform the diagnostic test themselves: 'I would perform the diagnostic test myself; the veterinarian does not have time for this; (FG 1, Farmer 3, male); 'I would perform the test myself. If the veterinarian performed the test, I would have to pay for his services again' (FG 4, Farmer 8, male).

\section{Discussion}

The current study aimed to explore the salient beliefs, and the biases that might distort these beliefs, underlying farmers' adoption intention of diagnostic tools. Therefore, the study complements insights from Theory of Planned Behaviour with insights from behavioural economics to get a more in-depth understanding in how those beliefs are formed. Besides, inductive analysis brought forward two additional rational factors, and biases that misconstrue these rational factors, underlying farmers' adoption intention. Below, we will discuss the results of our qualitative focus group studies in light of previous research and emphasize on the theoretical and practical implications of our research. See Appendix A, Fig. A1 for an overview of the results.

\subsection{Current habitual behaviour}

Most farmers reported experiencing problems with mange on their farms. These results are in line with the findings of Sarre et al. (2012), who established mange to be prevalent on $74 \%$ of the beef cattle farms in Flanders (affecting the Belgian Blue breed only). However, the severity of mange is subjective and varies between farmers. In particular, according to the descriptive results (see Table 1), only 10 farmers indicated high prevalence of mange on their farm $(>20 \%$ of their cattle infested) while the other farmers indicated moderate $(<20 \% ; 22$ farmers) or no $(0 \% ; 4$ farmers) prevalence of mange on their farm. Despite the perceived low or high prevalence of mange in their herd, the majority agreed that mange is a severe problem. Interestingly, although farmers perceive the disease to be severe, most of them also perceived mange as relatively controllable. The results of the present data suggest that this attitude can be attributed to farmers' rather positive attitudes towards acaricides (and in particular amitraz) to control mange on their farm. Importantly, attitudes towards current treatment methods are good predictors of farmers' behaviour when it comes to disease control management (Garforth et al., 2006). For example, Vande Velde et al. (2015) demonstrated that attitude towards anthelminthic drugs to control for GIN infections in dairy cattle hindered farmers' intention to adopt diagnostic tools to enable a more targeted use of anthelmintic drugs. As a consequence, we suggest that current habitual behaviour (specifically, farmers' rather positive attitude towards acaricides) can be seen as a barrier for intention to adopt diagnostic tools.

\subsection{Optimism Bias \& availability Bias distorting risk perception acaricide resistance}

The presence of macrocyclic lactone resistance on Belgian Blue beef farms has been established by Van Mol et al. (2020). The authors detected treatment failure with macrocyclic lactones on 12 out of 16 beef cattle farms in Flanders. Nonetheless, farmers were sceptical about their farm being susceptible to acaricide resistance in general (i.e., low perceived susceptibility) since they still believed in the efficacy of other acaricides besides macrocylic lactones to control mange. Similar results were found in a study by Vande Velde et al. (2018), wherein farmers' positive attitude towards anthelmintic treatments weakened dairy farmers' risk perception of anthelmintic resistance. Notably, risk perception is a subjective assessment and is formed by several factors such as psychological influences and personal experience (Slovic, 1999). As such, we suggest that the optimism bias and availability bias might have distorted farmers' subjective assessment of their farm being susceptible to acaricide resistance. The results demonstrated that farmers underestimated the probability of acaricide resistance occurring on their farm (i.e., optimism bias) mainly because of their positive attitude towards amitraz.

In addition, the availability bias can be linked to the optimism bias (Facione, 2002; Levi and Pryor, 1987). The results have shown that farmers relied on the incidence of mange on their farms (i.e., availability bias) to assess the judgement of acaricide resistance occurring on their farm. This availability bias might have led farmers to underestimate the probability of acaricide resistance occurring on their farm in the future (i.e., optimism bias). Particularly, farmers believed that implementing a new diagnostic tool would only be beneficial for farms with high prevalence of mange. Importantly, the descriptive results (see Table 1) showed that only 10 farmers perceived that more than $20 \%$ of their cattle was infested. Hence, we can assume that this availability bias is the least developed amongst the majority of the farmers. Moreover, Sarre et al. (2012) has demonstrated that farmers have misperceptions related to the prevalence of mange on their farm in that they underestimate the prevalence of mange in their herd (Sarre et al., 2012).

Consequently, farmers' low availability bias (i.e., the bias that is the least developed due to the underestimation of prevalence of mange; Sarre et al., 2012) may give rise to the optimism bias. Both biases may 
thus further weaken farmers' susceptibility to the problem (i.e., risk perception acaricide resistance). Importantly, when a perceived threat is appraised as trivial (i.e., low severity) or irrelevant (i.e., low susceptibility), there will be little motivation to respond to the threat (Maloney, Lapinski \& Witte, 2011). Consequently, we believe that farmers' risk perception towards acaricide resistance formed a barrier to farmers' adoption intention of diagnostic tools.

Although the availability bias and optimism bias has been found to influence farmers' risk perception related to experiencing negative climate change events (e.g., experiencing droughts; Dang et al., 2012; Duinen et al., 2015), this study adds to the literature that both biases can also play a role in farmers' disease control behaviours.

\subsection{Loss aversion Bias \& time discounting Bias distorting behavioural beliefs}

Farmers considered investing in diagnostic tools if they believed it would result in decreased costs (i.e., less money spent on current acaricides) and in long-term benefits exceeding the investment, which are both related to economic reasons. Similar results were found in a study on farmers' adoption of diagnostic tools in sustainable GIN control in dairy cattle (Vande Velde et al., 2018). Other studies also suggested that economic reasons (e.g., decreased costs, increased profitability) are important motivations for on-farm decision making (Alarcon et al., 2014; Borges et al., 2019; Jones et al., 2015; Ritter et al., 2017). However, when further analysing the data, we believe that the loss aversion bias and time discounting bias might have misconstrued this behavioural belief.

In particular, the present data suggests that the majority of the farmers tended to give more weight to the perceived losses resulting from adopting diagnostic tools (e.g., less profitability, monetary costs) than to the perceived benefits in the short and long term (e.g., decreased monetary costs, stop emerging acaricide resistance, long-term productivity). In reality, the benefits of adopting the diagnostic tool would outweigh the immediate costs, suggesting a mismatch between farmers' judgement and reality (i.e., behavioural bias). Accordingly, we suggest that both behavioural biases might have weakened the belief (for the farmers in question) that implementing diagnostic tools would result in positive economic outcomes. These findings are in line with previous literature in the agricultural domain suggesting that the loss aversion bias and time discounting bias influence farmers' decision-making (Dessart et al., 2019; Huijps et al., 2010).

In general, when farmers believed the diagnostic tool would be an effective means of optimising the control of mange (i.e., perceived usefulness), it motivated them to adopt the tool. Perceived usefulness refers to the degree to which a person believes that a new product/ technology would enhance outcomes such as job performance (Davis, 1989). The diagnostic tools' perceived usefulness can therefore be linked to decreased costs in time and labour, which can also be related to economic reasons (Borges et al., 2019). Furthermore, according to the Technology Acceptance Model (Davis, 1989), perceived usefulness forms an important predictor of attitudes toward a novel product.

Contribution to animal health also motivated farmers to adopt diagnostic tools on their farms. This is in line with previous studies suggesting that animal health is an important behavioural factor in the adoption of preventive measures on farms or participation in treatment programs to increase animal welfare (Borges et al., 2019; Velde et al., 2018). Increased animal welfare can also be seen as an indirect economic factor (e.g., increase profitability) or an ethical factor (e.g., decrease animal suffering) that influences farmers' decision-making (Borges et al., 2019; Hansson and Lagerkvist, 2015; Velde et al., 2018). With regard to indirect economic factors, mange induces intense pruritus, and these pathological changes have a significant effect on the animal's metabolism and accordingly, its growth (Sarre et al., 2012). Mange can therefore lead to a decrease in profitability in the beef industry (Rehbein et al., 2016). With regard to ethical factors, besides the economic consequences, mange is also a serious welfare issue, as it is a severe debilitating skin condition causing the animals to suffer (Lekimme et al., 2010). The results of our study have shown that farmers were motivated to adopt diagnostic tools for both economic reasons (i.e., more profitability) and ethical reasons (e.g., less stress and suffering of the animals).

\subsection{Bandwagon effect Bias distorting normative beliefs}

As shown in the present data, the two important referents that can place pressure upon farmers' intention to adopt a diagnostic tool are veterinarians and farmers colleague. We believe that veterinarians are the most important normative referent (although this belief was not quantitatively measured in a survey) since veterinarians' opinions are valued most for the adoption of diagnostic tools on beef cattle farms. Many other studies in the agricultural domain also suggest that veterinarians are an important normative factor, driving subjective norms (Borges et al., 2019; Jones et al., 2015; Sok et al., 2015; Speksnijder and Wagenaar, 2018).

Importantly, it has been suggested in the literature that veterinarians have been found to often fall short in motivating farmers to change treatment practices, such as reducing antibiotic usage (Alarcon et al., 2014; Jones et al., 2015; Laanen et al., 2014). This trend was also noticeable in our data as farmers claimed there was a lack of communication about acaricide resistance from their veterinarian. Furthermore, farmers also valued the opinion and behaviour of other farmers related to adopting the diagnostic tool. We believe that the bandwagon effect bias might have distorted this normative belief. In particular, several farmers were inclined to follow the behaviour of other farmers, which is referred to in the literature as descriptive norms - i.e., what the majority is doing (Cialdini and Trost, 1998). The bandwagon effect bias refers to the tendency to follow the opinion or behaviour adopted by the majority, or in other words, refers to conformism to descriptive norms (Samson, 2016). Thus this bias might have strengthened the belief that farmers value the opinion of their colleague-farmers. This is in line with previous literature suggesting that fellow farmers are perceived to be the most influential referent underlying farmers' perceived descriptive and injunctive (or subjective) norms (Ritter et al., 2017; Sok et al., 2015). Furthermore, perceived social norms, comprised of descriptive norms and injunctive norms (or subjective norms) within a farmers' community, have been found to have a strong influence on farmers' intention to adopt preventive measures related to disease control (Alarcon et al., 2014; Ellis-Iversen et al., 2011; Ritter et al., 2017).

However, not all farmers claimed to follow the behaviour of their colleagues. We suggest that farmers' personality traits might also have influenced their intention to adopt a diagnostic tool (Sok et al., 2018). Taken into account the five clusters of personality traits (i.e., the Big Five: extraversion, openness to new experiences, agreeableness, neuroticism and conscientiousness; McCrae and Costa, 1992), openness and consciousness may have been related to farmers' willingness to implement the test first (Sok et al., 2018). Openness refers to the motivation to seek new experiences through curiosity and the preference for creativity or variety. Consciousness refers to the desire to do a task well and to take obligation towards others seriously (McCrae and Costa, 1992). Importantly, as personality traits are measured by standardized scales (McCrae and Costa, 1992), we can only suggest that famers' personality traits could form a boundary condition for the normative influence of colleague-farmers (Dessart et al., 2019). Future studies could explore the possible role of Belgian Blue cattle farmers' personality trait (measured with standardized scales) in their intention to adopt diagnostic tools.

\subsection{Loss aversion Bias distorting control beliefs}

Farmers' perceptions of the economic costs of the diagnostic tool tended to hamper their intention to adopt such tools. This confirms previous literature on farmers' disease control behaviours, which 
suggests that farmers' perceptions of the feasibility of the behaviour (e. g., costs, labour) greatly influences their intentions to implement disease control measures (Ritter et al., 2017).

Interestingly, as the data revealed that the diagnostic tools' perceived economic costs were at the forefront of farmers' thoughts, we believe that the loss aversion bias might have misconstrued this control belief. In particular, the majority of the farmers tended to focus more on the perceived losses (i.e., economic costs) related to adopting a diagnostic tool than to the benefits of the same magnitude (e.g., decreased costs spent on current acaricides). Consequently, besides distorting behavioural beliefs (i.e., economic reasons), we believe that this loss aversion bias also distorted farmers' objective measures of perceptions of costs and benefits related to the diagnostic tool. Hence, the loss aversion bias might have strengthened the belief that economic costs related to the diagnostic tool formed an impeding factor for farmers' adoption intention.

For half of the farmers, the veterinarians' assistance was found to be a facilitating factor to use the diagnostic tool on their farm. This might be linked to farmers' beliefs in their ability to perform the test themselves. In particular, for farmers who believed they were not able to do so, the role of the veterinarian should be considered to stimulate the uptake of the diagnostic tool.

\subsection{Limitations and further research}

As with all research, the current study has some limitations. First, before the focus groups were conducted, the researchers made sure that there was a minimum of six and a maximum of 12 participants in the focus groups. However, for one focus group (Antwerp), three participants did not show up.

Secondly, the biases could be explored more thoroughly in future research. Particularly, the current biases are situated at the level of farmers' adoption intention (and not actual adoption), since a diagnostic tool that tests for acaricide resistance is not yet available on the market. Therefore, we gained few insights about the factors/biases related to farmers' actual adoption. If the diagnostic tool became available on the market, future studies could explore whether the biases that came forward in this study reappear in farmers' minds when actually deciding to adopt such tools. This article discussed the presence of five behavioural biases in farmers' thoughts; however, there are many more biases to discover, which could provide interesting venues for future research.

Third, since mange is only a major problem in Belgium, affecting the Belgian Blue breed in particular (this breed is predominantly present in Belgium), the research population is limited to Belgian Blue cattle farmers. Therefore the results cannot be extrapolated to all cattle farmers in Belgium or abroad. In addition, it is possible that only the more engaged farmers participated in the focus group studies, which makes the collected data vulnerable to selection bias. However, in Belgium, there are approximately 3100 Belgian Blue cattle farmers (Danckaert et al., 2017), and $99 \%$ of beef cattle in Belgium consists of the Belgian Blue breed. Consequently, there unlikely to be major selection bias, since the largest represented beef cattle breed in Belgium is the Belgian Blue breed.

Fourth, in order to minimise the observation bias, after conducting the focus group studies and analysing the data, findings were discussed amongst the authors present on the focus group studies. Besides, the results presented in this paper were also crosschecked by the moderator (i.e., veterinary scientist) of the focus group studies. Finally, the focus groups were only audio recorded; therefore, it was impossible to accurately to discuss the non-verbal interaction of participants with the other group members in-depth. This limitation might not have occurred when individual interviews would have been conducted.

Although this study provided in-depth insight into the salient beliefs, and the biases that distort these beliefs, underlying farmers' adoption intention, future studies could use these beliefs in a quantitative study to measure behavioural, normative and control beliefs (i.e., to quantify the importance of each belief). Accordingly, one could explore the explained variance of each belief for each determinant of the TPB (Borges et al., 2019). Given that risk perception of acaricide resistance was found to be an important inhibiting factor for adoption intention that arose from this data, and given that this study is one of the few studies that has explored farmers' risk perception related to this resistance, future studies could further elaborate on this. More specifically, future studies could quantitatively (e.g., by means of a survey) investigate farmers' risk perceptions of acaricide resistance and explore how this may influence farmers' adoption intentions. Future (qualitative) studies could also explore veterinarians' knowledge and beliefs about acaricide resistance and their beliefs (and possible related biases) about diagnostic tools, since veterinarians are considered highly important for the decision to implement new treatment methods. This might offer insights for communication strategies to motivate veterinarians to recommend diagnostic tools on beef cattle farms.

\section{Policy implications}

Some important recommendations to promote the uptake of diagnostic tools can be distilled from the findings above. These recommendations can be used by policy makers to develop effective communication strategies; they can also be used by veterinarians in their advisory role of influencing farmers' intention to adopt diagnostic tools. As several biases tended to distort farmers' rational thinking, communication strategies should de-bias (i.e., designed to eliminate biases; this can be done by activating farmers' deliberate reasoning) or should exploit these biases (i.e., using biases to shift behaviour in the desired direction) to stimulate the uptake of diagnostic tools (Dessart et al., 2019; Samson, 2016).

First, there is a need to increase farmers' risk-perception towards acaricide resistance, as this formed a barrier to farmers' adoption intention. Considering the importance farmers attach to veterinarians' opinions and the lack of information about resistance provided by their veterinarians, veterinarians should act as the main information source for farmers on the emerging acaricide resistance. Furthermore, communication strategies should exploit the availability bias and counteract the optimism bias. For example, messages could incorporate accurate numbers of prevalence of mange on farms (e.g., on $70 \%$ of Belgian Blue cattle farms, more than $30 \%$ of the animals are infested with P. ovis mites; Sarre et al., 2012). Countering the optimism bias can also be done through education or information provision on acaricide resistance (e.g., what it is, what is causing it, how to prevent it, how diagnostic tools can prevent emerging acaricide resistance, the role of the veterinarian).

Second, communication strategies should counter the loss aversion bias and time discounting bias, as these tended to weaken farmers' belief that the diagnostic tool would increase profitability (i.e., behavioural belief). This can be done by activating farmers' deliberate reasoning by emphasizing the benefits of implementing diagnostic tools on farms (e. g., decreased costs in money and time, return on investment). These messages could also highlight the perceived usefulness of the diagnostic tool and its contribution to animal wellbeing.

Third, the results of this study suggest that farmers are motivated to adopt the diagnostic tool when they believe it is recommended by their veterinarian. The default bias refers to the fact that individuals have a preference for things to remain as they are (i.e., default setting), because any change can be perceived as a loss. As such, in situations where choices are difficult, the default option may be perceived as the recommended course of action. Accordingly, the veterinarian has an important role in exploiting this default bias, in that (s)he should offer the diagnostic tool as a default treatment. Farmers will follow this default option, resulting in uptake of the diagnostic tool.

Communication strategies could also emphasize that the majority of Belgian Blue cattle farmers have adopted the diagnostic tool or have a positive opinion about it (i.e., descriptive norms, bandwagon effect 


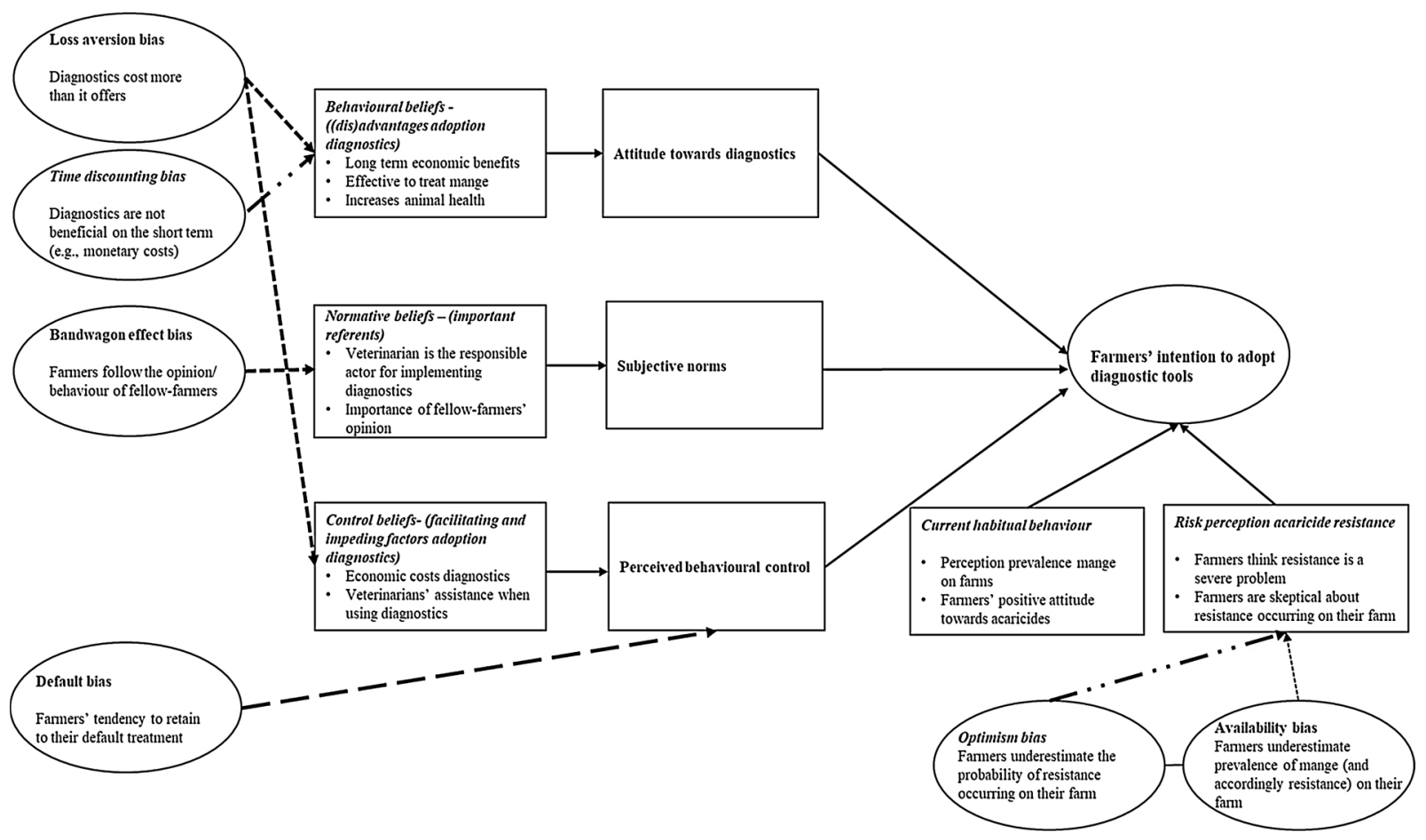

Fig. A1. Visual presentation of the current study's findings, combined with findings of the conceptual model of Mingolla et al. (2019). Note. The dotted lines represent the biases that distort the rational factors (i.e., salient beliefs and risk perception). The new constructs, gathered from the focus groups, are indicated in Italic. The biases that confirm findings from the previous study are indicted in dotted thick lines. The new biases that came forward during the focus groups are indicated in line-dotted-line-dotted lines. The biases different from the previous study are indicated.

bias). Finally, countering the loss aversion bias could also facilitate the adoption of diagnostic tools (i.e., control belief). As for the majority of the farmers, veterinarian assistance was anticipated to facilitate the adoption of diagnostic tools, the role of the veterinarian should be emphasized in communication strategies. Incorporating these findings will ultimately motivate farmers to adopt diagnostic tools, enabling a more sustainable use of acaricides, resulting in sustainable mange control in general.

\section{Conclusion}

Our findings suggest that farmers' intention to adopt diagnostic tools is not entirely based on rational deliberation (i.e., formation of beliefs and risk perception) but is also affected by other irrational factors, such as behavioural biases. Insights into these biases are important as they might strengthen a belief negatively related to the desired behaviour or weaken a belief positively related to the desired behaviour The results of our study indeed show that farmers' (mis)perceptions of cost and benefits related to diagnostics (i.e., loss aversion bias and time discounting bias) strengthen the belief that the cost of the diagnostic tool would form an impeding factor (i.e., behavioural and control belief). Conversely, the perception that other farmers would use the diagnostic tool (i.e., descriptive norms, bandwagon effect bias) strengthens their normative beliefs. Besides, our study demonstrates that farmers' risk perception (and in particular; perceived susceptibility) is misaligned with the reality since farmers underestimate the prevalence of mange, and accordingly, acaricide resistance on their farm (i.e., the availability bias and optimism bias).

Importantly, this paper identified rational and behavioural biases underlying farmers' intention to adopt a specific behaviour in a specific agricultural context (i.e., adopting disease control measures). Thus, we may not conclude that these factors apply for other behaviours, such as implementing environmentally sustainable practices or other disease control programs.

\section{Acknowledgements}

We would like to thank all the farmers who participated in our focus group studies and provided us with the data. Thanks to Animal Health Care Flanders, Belgium, for providing us with an up-to-date data list of all Belgian Blue cattle farmers. This research was funded by [Ghent University, BelgiumBOF24J2016000402 and Research FoundationFlanders, BelgiumFWO.3E0.2015.0035.01].

\section{Appendix A}

\section{References}

Ajzen, I., 1991. The theory of planned behavior. Organ. Behav. Hum. Decis. Process. 50 (2), 179-211.

Ajzen, I., 2002. Constructing a TPB Questionnaire: Conceptual and Methodological Considerations.

Alarcon, P., Wieland, B., Mateus, A.L., Dewberry, C., 2014. Pig farmers' perceptions, attitudes, influences and management of information in the decision-making process for disease control. Prev. Vet. Med. 116 (3), 223-242.

Bagheri, A., Bondori, A., Allahyari, M.S., Damalas, C.A., 2019. Modeling farmers' intention to use pesticides: an expanded version of the theory of planned behavior. J. Environ. Manage. 248, 109291.

Blutke, A., Börjes, P., Herbach, N., Pfister, K., Hamel, D., Rehbein, S., Wanke, R., 2015. Acaricide treatment prevents adrenocortical hyperplasia as a long-term stress reaction to psoroptic mange in cattle. Vet. Parasitol. 207 (1-2), 125-133.

Borges, J.A.R., Tauer, L.W., Lansink, A.G.O., 2016. Using the theory of planned behavior to identify key beliefs underlying Brazilian cattle farmers' intention to use improved natural grassland: a MIMIC modelling approach. Land Use Policy 55, 193-203.

Borges, J.A.R., de Faria Domingues, C.H., Caldara, F.R., da Rosa, N.P., Senger, I., Guidolin, D.G.F., 2019. Identifying the factors impacting on farmers' intention to adopt animal friendly practices. Prev. Vet. Med. 170, 104718.

Camerer, C.F., Loewenstein, G., Rabin, M., 2004. Advances in Behavioral Economics, Princeton university press.

Cialdini, R.B., Trost, M.R., 1998. Social Influence: Social Norms, Conformity and Compliance.

Conner, M., Sparks, P., 2005. Theory of planned behaviour and health behaviour. Predicting health behaviour 2 (1), 121-162. 
da Silva, J.R., Mauad, J.R.C., de Faria Domingues, C.H., Marques, S.C.C., Borges, J.A.R., 2020. Understanding the intention of smallholder farmers to adopt fish production. Aquac. Rep. 17, 100308.

Davis, F.D., 1989. Perceived usefulness, perceived ease of use, and user acceptance of information technology. Mis Q. 319-340.

Daxini, A., O’Donoghue, C., Ryan, M., Buckley, C., Barnes, A.P., Daly, K., 2018. Which factors influence farmers' intentions to adopt nutrient management planning? J. Environ. Manage. 224, 350-360.

Dessart, F.J., Barreiro-Hurlé, J., van Bavel, R., 2019. Behavioural factors affecting the adoption of sustainable farming practices: a policy-oriented review. Eur. Rev. Agric. Econ. 46 (3), 417-471.

Doherty, E., Burgess, S., Mitchell, S., Wall, R., 2018. First evidence of resistance to macrocyclic lactones in Psoroptes ovis sheep scab mites in the UK. Vet. Rec. 182 (4), 106-106.

Ellis-Iversen, J., Smith, R.P., Gibbens, J.C., Sharpe, C.E., Dominguez, M., Cook, A.J.C., 2011. Risk factors for transmission of foot-and-mouth disease during an outbreak in southern England in 2007. Vet. Rec.

Facione, N.C., 2002. Perceived risk of breast cancer: influence of heuristic thinking. Cancer Pract. 10 (5), 256-261.

Frederiks, E.R., Stenner, K., Hobman, E.V., 2015. Household energy use: applying behavioural economics to understand consumer decision-making and behaviour. Renewable Sustainable Energy Rev. 41, 1385-1394.

Furnham, A., 1986. Response bias, social desirability and dissimulation. Pers. Individ. Dif. 7 (3), 385-400.

Hansson, H., Lagerkvist, C.J., 2015. Identifying use and non-use values of animal welfare: evidence from Swedish dairy agriculture. Food Policy 50, 35-42.

He, R., Jin, J., Gong, H., Tian, Y., 2019. The role of risk preferences and loss aversion in farmers' energy-efficient appliance use behavior. J. Clean. Prod. 215, 305-314.

Huijps, K., Hogeveen, H., Lam, T.J., Lansink, A.O., 2010. Costs and efficacy of management measures to improve udder health on Dutch dairy farms. J. Dairy Sci. 93 (1), 115-124.

Hyland, J.J., Heanue, K., McKillop, J., Micha, E., 2018. Factors underlying farmers' intentions to adopt best practices: the case of paddock based grazing systems. Agric. Syst. 162, 97-106.

Jansen, J., Lam, T.J., 2012. The role of communication in improving udder health. Veterinary Clinics: Food Animal Practice 28 (2), 363-379.

Jones, P.J., Marier, E.A., Tranter, R.B., Wu, G., Watson, E., Teale, C.J., 2015. Factors affecting dairy farmers' attitudes towards antimicrobial medicine usage in cattle in England and Wales. Prev. Vet. Med. 121 (1-2), 30-40.

Kahneman, D., Slovic, S.P., Slovic, P., Tversky, A., 1982. Judgment Under Uncertainty: Heuristics and Biases. Cambridge university press.

Kitzinger, J., 1995. Qualitative research: introducing focus groups. BMJ 311 (7000), 299-302. https://doi.org/10.1136/bmj.311.7000.299.

Korteling, J.E., Brouwer, A.-M., Toet, A., 2018. A neural network framework for cognitive bias. Front. Psychol. 9, 1561.

Laanen, M., Maes, D., Hendriksen, C., Gelaude, P., De Vliegher, S., Rosseel, Y., Dewulf, J., 2014. Pig, cattle and poultry farmers with a known interest in research have comparable perspectives on disease prevention and on-farm biosecurity. Prev. Vet. Med. 115 (1-2), 1-9.

Lekimme, M., Farnir, F., Marechal, F., Losson, B., 2010. Failure of injectable ivermectin to control psoroptic mange in cattle. Vet. Rec. 167 (15), 575-576. https://doi.org/ 10.1136/vr.c4906.

Levi, A.S., Pryor, J.B., 1987. Use of the availability heuristic in probability estimates of future events: the effects of imagining outcomes versus imagining reasons. Organ. Behav. Hum. Decis. Process. 40 (2), 219-234.

Lifschitz, A., Fiel, C., Steffan, P., Cantón, C., Muchiut, S., Dominguez, P., Lanusse, C., Alvarez, L., 2018. Failure of ivermectin efficacy against Psoroptes ovis infestation in cattle: integrated pharmacokinetic-pharmacodynamic evaluation of two commercial formulations. Vet. Parasitol. 263, 18-22.

McCrae, R.R., Costa Jr., P.T., 1992. Discriminant validity of NEO-PIR facet scales. Educ. Psychol. Meas. 52 (1), 229-237.

McLeod, L.J., Hine, D.W., Please, P.M., Driver, A.B., 2015. Applying behavioral theories to invasive animal management: towards an integrated framework. J. Environ. Manage. 161, 63-71.
Mingolla, C., Hudders, L., Vanwesenbeeck, I., Claerebout, E., 2019. Towards a biased mindset: an extended Theory of Planned Behaviour framework to predict farmers intention to adopt a sustainable mange control approach. Prev. Vet. Med. 169, 104695.

Mullainathan, S., Thaler, R.H., 2000. Behavioral Economics. National Bureau of Economic Research.

OECD, 2012. Farmer Behaviour, Agricultural Management and Climate Change. OECD. https://doi.org/10.1787/9789264167650-en.

Rehbein, S., Visser, M., Meyer, M., Lindner, T., 2016. Ivermectin treatment of bovine psoroptic mange: effects on serum chemistry, hematology, organ weights, and leather quality. Parasitol. Res. 115 (4), 1519-1528.

Rezaei, Rohollah, Mianaji, S., Ganjloo, A., 2018. Factors affecting farmers' intention to engage in on-farm food safety practices in Iran: extending the theory of planned behavior. J. Rural Stud. 60, 152-166.

Rezaei, Rohollah, Seidi, M., Karbasioun, M., 2019. Pesticide exposure reduction: extending the theory of planned behavior to understand Iranian farmers' intention to apply personal protective equipment. Saf. Sci. 120, 527-537.

Ritter, C., Jansen, J., Roche, S., Kelton, D.F., Adams, C.L., Orsel, K., Erskine, R.J., Benedictus, G., Lam, T.J., Barkema, H.W., 2017. Invited review: determinants of farmers' adoption of management-based strategies for infectious disease prevention and control. J. Dairy Sci. 100 (5), 3329-3347.

Samson, A., 2016. The Behavioral Economics Guide 2016 (with an Introduction by Gerd Gigerenzer). Behavioral Science Solutions Ltd.

Sarre, C., De Bleecker, K., Deprez, P., Levecke, B., Charlier, J., Vercruysse, J., Claerebout, E., 2012. Risk factors for Psoroptes ovis mange on Belgian Blue farms in Northern Belgium. Vet. Parasitol. 190 (1-2), 216-221.

Sarre, C., González-Hernández, A., Van Coppernolle, S., Grit, R., Grauwet, K., Van Meulder, F., Chiers, K., Van den Broeck, W., Geldhof, P., Claerebout, E., 2015. Comparative immune responses against Psoroptes ovis in two cattle breeds with different susceptibility to mange. Vet. Res. 46 (1), 131.

Shepperd, J.A., Carroll, P., Grace, J., Terry, M., 2002. Exploring the causes of comparative optimism. Psychol. Belg. 42 (1/2), 65-98.

Sok, J., Hogeveen, H., Elbers, A.R.W., Lansink, A.O., 2015. Farmers' beliefs and voluntary vaccination schemes: bluetongue in Dutch dairy cattle. Food Policy 57, $40-49$.

Sok, J., Hogeveen, H., Elbers, A.R.W., Oude Lansink, A., 2018. Perceived risk and personality traits explaining heterogeneity in Dutch dairy farmers' beliefs about vaccination against Bluetongue. J. Risk Res. 21 (5), 562-578.

Speksnijder, David C., Wagenaar, J.A., 2018. Reducing antimicrobial use in farm animals: how to support behavioral change of veterinarians and farmers. Anim. Front. 8 (2), 4-9.

Strauss, A., Corbin, J., 1994. Grounded theory methodology. Handbook of qualitative research $17,273-285$.

Stromberg, P.C., Fisher, W.F., Guillot, F.S., Pruett, J.H., Price, R.E., Green, R.A., 1986 Systemic pathologic responses in experimental Psoroptes ovis infestation of Hereford calves. Am. J. Vet. Res. 47 (6), 1326.

Thomas, D.R., 2006. A general inductive approach for analyzing qualitative evaluation data. Am. J. Eval. 27 (2), 237-246.

van Dijk, W.F., Lokhorst, A.M., Berendse, F., De Snoo, G.R., 2016. Factors underlying farmers' intentions to perform unsubsidised agri-environmental measures. Land Use Policy 59, 207-216.

van Mol, W., De Wilde, N., Casaert, S., Chen, Z., Vanhecke, M., Duchateau, L., Claerebout, E., 2020. Resistance against macrocyclic lactones in Psoroptes ovis in cattle. Parasit. Vectors 13 (1), 1-9.

Velde, F.V., Charlier, J., Hudders, L., Cauberghe, V., Claerebout, E., 2018. Beliefs, intentions, and beyond: a qualitative study on the adoption of sustainable gastrointestinal nematode control practices in Flanders' dairy industry. Prev. Vet. Med. 153, 15-23.

Wolf, C.A., 2017. Using institutional and behavioural economics to examine animal health systems. Rev. - Off. Int. Epizoot. 36 (1), 67-76.

Yuriev, A., Dahmen, M., Paillé, P., Boiral, O., Guillaumie, L., 2020. Pro-environmental behaviors through the lens of the theory of planned behavior: a scoping review. Resour. Conserv. Recycl. 155, 104660. 